\title{
Inhibition in Selective Attention
}

\author{
Dirk van Moorselaar ${ }^{1,2,{ }^{*}}$ \& Heleen A. Slagter ${ }^{1,2, *}$
}

1. Department of Experimental and Applied Psychology, Vrije Universiteit Amsterdam, the Netherlands

2. Institute of Brain and Behaviour Amsterdam, the Netherlands

* Both authors equally contributed to this work.

Keywords: attention; inhibition; predictive processing; brain; EEG

Number of pages: 32

Number of Figures: 2

\section{Acknowledgements}

This work was supported by a European Research Council (ERC) starting grant (679399) to H.A.S.

Please address correspondence to:

Dr. Dirk van Moorselaar and/or Prof. Dr. Heleen Slagter

Department of Experimental and Applied Psychology

Vrije Universiteit Amsterdam

e-mail: dirkvanmoorselaar@gmail.com or h.a.slagter@vu.nl 


\section{Abstract}

Our ability to focus on goal-relevant aspects of the environment is critically dependent on our ability to ignore or inhibit distracting information. One perspective is that distractor inhibition is under similar voluntary control as attentional facilitation of target processing. However, a rapidly growing body of research shows that distractor inhibition often relies on prior experience with the distracting information or other mechanisms that need not rely on active representation in working memory. Yet, how and when these different forms of inhibition are neurally implemented remains largely unclear. Here, we review findings from recent behavioral and neuroimaging studies to address this outstanding question. We specifically explore how experience with distracting information may change the processing of that information in the context of current predictive processing views of perception: by modulating a distractor's representation already in anticipation of the distractor, or after integration of top-down and bottom-up sensory signals. We also outline directions for future research necessary to enhance our understanding of how the brain filters out distracting information. 


\section{Introduction}

Our daily visual surroundings, like city crossroads, contain a multitude of objects, most of which are irrelevant to our current goals. To flexibly navigate such complex environments, ignoring visual distractions (e.g., advertisement billboards) is arguably equally important as focusing on goal-relevant information (e.g., surrounding cars). Yet, while over the past several decades, much has been learned about how selective attention can facilitate neural processing of goal-relevant information (1-3), the mechanisms that underlie suppression of visual distractions at the neural level remain relatively poorly understood.

Facilitation and inhibition have long been envisioned as two sides of the same coin $(4,5)$. Yet, a rapidly growing body of work indicates that distractor suppression is not unitary and reveals itself in different guises, likely reflecting multiple underlying neural architectures and processes $(6,7)$. This diversity is also hard to reconcile with influential attentional theories (e.g., biased competition) that only regard suppression as resulting from inhibitory influences arising from competitive interactions between neural populations $(1,8)$. Instead, it is now evident that inhibition can also be accomplished through neural mechanisms that are (in part) independent from well-characterized facilitative attention mechanisms $(6,9,10)$.

Here, we review recent work on the neural mechanisms underlying inhibition in selective attention, and specifically address the outstanding question: how and under what conditions can the brain, if at all, suppress distractors before they capture attention? In this, we dissociate between proposed preparatory mechanisms that suppress distractor features in advance $(9,11,12)$ and suppressive mechanisms that come about in response to distracting sensory input $(7,10,13)$. While the long-standing view has been that inhibition of distracting or irrelevant sensory information is under direct, volitional control similar to attention to goalrelevant aspect of the environment, it is becoming increasingly clear that suppression is not under direct topdown control and can be implemented via a multitude of underlying mechanisms, each tuned to specific circumstances $(6,9,14)$. Recent behavioral studies, for example, have shown that the ability to ignore distracting information often strongly depends on learning based on previous experiences with the distracting information $(15,16)$. Yet, what factors determine the ability to inhibit distractor information and which specific set of neural mechanisms arise under different circumstances remain topics of active debate $(6,9,10,14)$.

In addressing this outstanding issue, we will discuss evidence from key behavioral and neuroimaging studies in humans and some animal studies in light of different ideas on distractor inhibition $(6,9,10,17)$. The 
picture that emerges from this is that how the brain deals with distracting information is not simply determined by whether or not information is relevant vs. irrelevant to the task at hand, but influenced by many factors, including previous experience with the distracting information (expectations), whether suppression is feature based or space based, target-distractor similarity, the level at which distracting information can be suppressed, and distractor salience. Building on the notion of expectation-dependent distractor suppression (9), we specifically discuss different ways in which experience with distracting information may change the neural representation and processing of that information in the context of current predictive processing accounts of perception $(18,19)$. In these accounts, expectations derived from past experience and grounded in statistical regularities in the environment strongly shape sensory information processing and thereby perception, but it is unclear if and how predictive processing is affected by the distracting or irrelevant nature of encountered information. Expectations could result in tuning towards or away from the expected distractor features (Figure 1A-B), or they could not be expressed in changes in anticipatory tuning, but exert their effects via synaptic plasticity (Figure 1C). We will also discuss recent findings that show that alpha-band oscillations may play an important role in facilitating processing of goal-relevant information, by suppressing noise (20) or stabilizing the representation of the attended stimulus $(21,22)$. We explore the possibility that alpha oscillations could similarly modulate the representation of distracting information. We end by discussing important avenues for future research.

\section{Preparatory distractor suppression}

It is currently actively debated whether distractor inhibition is under similar flexible top-down control as target facilitation and depends on representation in working memory $(6,9,14)$. A large body of work shows a close relationship between selective attention and visual working memory, such that attention is directed towards visual information that is actively represented in working memory $(1,23)$. Indeed, many studies have demonstrated that the content of working memory automatically biases attention towards matching incoming information, even when that information is known to be detrimental to performance at the current task (24, 25). It has been proposed that information in working memory about anticipated distracting input can also be represented as a 'rejection template' such that matching input will be strategically inhibited $(11,12)$. In contrast to the notion of direct working memory-based distractor suppression, it has also been suggested that 
suppression of distracting information may rely on mechanisms that render the representation of the target in working memory maximally distinct from that of the distractor, thereby indirectly decreasing the chance of distractor selection $(17,26)$. In yet another account, distractor inhibition may bypass representation in working memory altogether, relying on expectation-dependent suppression (9). In the below, we discuss these different accounts of preparatory inhibition in more detail in light of the empirical literature to address the outstanding question if distractor inhibition can be implemented in advance, and if so, how and under what conditions?

\section{Direct preparatory distractor suppression}

It has been proposed that information in working memory about anticipated distracting input can be represented as a 'rejection template' such that matching distracting input will be strategically inhibited $(11,12$, 14). This notion of direct top-down attentional inhibition is challenged by behavioral studies showing that cues informing about the upcoming distractor location or feature carry no behavioral benefit $(15,27,28)$ or even hamper performance, when this information varies from trial to trial (29). These findings suggest that if anything, distractor templates in working memory result in increased distractibility and a prerequisite for successful preparatory inhibition may therefore be that the underlying mechanisms bypass working memory maintenance. However, there is also evidence that distractor foreknowledge can actually be used to selectively avoid or inhibit matching distractors $(11,12,30,31)$ or specific distractor locations $(32,33)$. Based on behavioral measures alone, however, it is hard to establish if these observed behavioral benefits of distractor foreknowledge are indeed driven by an advance inhibitory template or instead rely on a post-distractor inhibition mechanism ( 7 , 13). To unequivocally establish the existence of inhibitory templates and direct, active preparatory suppression, we need to turn to measures of brain activity. The dominant view in the cognitive neuroscience literature over the past two decades or so has been that just like a frontoparietal control network can enhance processing of goal-relevant information by biasing task-relevant sensory regions in advance, this control network can also inhibit sensory processing of distracting information in advance, and that alpha-band oscillations implement this top-down attentional bias $(4,34)$.

It is specifically proposed that alpha-band oscillations implement direct, top-down inhibition via the suppression of activity of sensory regions representing the task-irrelevant or distracting information $(4,34)$. Indeed, alpha oscillatory activity has been functionally linked to reduced cortical excitability (35) and there is 
abundant evidence from spatial attention studies that pre-stimulus alpha-band activity decreases over visual regions contralateral to attended target locations and/or increases over ipsilateral visual regions that represent the task-irrelevant hemifield (36-39). Several studies have also related increases in pre-stimulus alpha-band activity to anticipatory suppression of non-spatial visual features (40-42). These modulations in anticipatory alpha activity have generally been taken to reflect release of inhibition of task-relevant visual networks and enhanced inhibition of task-irrelevant visual networks. However, as also pointed out recently by Foster and Awh (43), evidence in support of the notion that alpha oscillations implement active, top-down distractor suppression is ambiguous. That is, the vast majority of attention studies reporting increased preparatory alpha activity over irrelevant visual regions used task designs in which foreknowledge about the distractor location or features was accompanied by foreknowledge about the target location or features, such as bilateral displays (36-38), rendering it possible that observed effects could also simply reflect attending away or secondary inhibition related to attention to the target (9). The same is true for studies that used cues that signaled the likelihood of upcoming distraction, but also provided information about the upcoming target, and examined changes in other measures of preparatory activity, such as the cue-evoked LDAP ERP component $(44,45)$.

To unambiguously establish a role for alpha-oscillations in preparatory inhibition it is not only important that the process of interest is selectively manipulated, but also that this cannot lead to changes in how attention is directed towards task-relevant information. In the spatial domain, one approach is to cue participants about the upcoming distracting information without making target information explicit, which could be accomplished by using visual search tasks with multiple stimulus locations. Of the substantial body of work linking alpha-band activity to spatial suppression, however, only a single study actually examined alpha-modulations in response to distractor location cues that were not also informative about the upcoming target location (46). In this study, pre-stimulus alpha-band activity tracked the anticipated location when the target was cued, but not when the distractor was cued. Rather than reflecting direct top-down inhibition, observed changes in pre-stimulus alpha activity reported previously could thus also be a secondary consequence of top-down target facilitation.

Although alpha oscillations have predominantly been linked to spatially specific suppression, as noted above, there is also evidence that alpha-suppression mechanisms operate in much the same manner during nonspatial selection (40-42). However, here too, observed effects cannot be unambiguously interpreted as active preparatory inhibition because knowledge about the upcoming irrelevant non-spatial feature was also always accompanied by knowledge about the relevant non-spatial feature. Moreover, the one study to date that 
specifically modulated distractor interference and examined whether alpha oscillations contribute to featurebased, preparatory distractor suppression investigated changes in alpha activity with respect to the location of the distractor stimulus. In this study by de Vries et al. (2019)(47), lateralized posterior alpha power did not dissociate between laterally presented colors in memory, which were cued to either be a target or a distractor in subsequent searches, further arguing against the idea that human observers can set up an inhibitory template in advance. Yet, although lateralized alpha power did not help to dissociate between targets and distractors, overall non-lateralized power over visual cortex was higher when observers were anticipating a distractor versus a target (see also (48)). Similarly, using $\mathrm{fMRI}$, another study observed less activation in large parts of visual cortex in response to distractor color cues relative to neutral or target cues (49), without this decrease being distinctive for specific distractor features (50). Together, these studies suggest that behavioral benefits observed as a function of distractor foreknowledge may be related to non-specific suppression of sensory activity, possibly in an attempt to filter out anticipated distractors. Yet, they do not support the notion of direct top-down preparatory distractor feature-specific inhibition.

The previous suggests that distractor suppression may rely on non-specific sensory inhibition. Indeed, benefits elicited by target location cues that concurrently signal distractor probability are increased on high relative to low probable distractor trials (51-53). In such contexts, when visual distraction is expected, but information about the upcoming distractor is non-specific, the visual system may prepare to inhibit perceptual processing as whole via increased posterior alpha power (48). Also, in a more recent fMRI study, preparatory BOLD activity increased in the middle frontal gyrus in frequent relative to infrequent distractor blocks, a signal increase that was subsequently accompanied by attenuation of signal processing in occipital cortex (54). Together, these latter studies suggest that non-specific suppressive mechanisms can be flexibly induced in anticipation of distractors, although the neural mechanisms underlying this form of suppression remain largely speculative. Alternatively, knowing that external distraction is likely to occur can simply also modulate arousal or response readiness, and affect performance through mechanisms unrelated to sensory inhibition per se. Future neuroimaging studies are necessary to determine how non-specific inhibition facilitates performance.

Thus, to date there is very little neural evidence in support of the notion that distractor foreknowledge is associated with direct top-down inhibition of activity in sensory regions representing the anticipated distractor location or feature. This may suggest, as discussed in the below, that distractor suppression relies on mechanisms that bypass working memory representation or only becomes evident after integration of bottom- 
up sensory input with top-down influences. However, given that only a handful of studies examined markers of active preparatory suppression, it is premature to conclude that direct top-down inhibition in either the spatial or feature domain is not possible. One intriguing possibility is that in contrast to pre-activating an attentional template, preparatory suppression is cognitively demanding and therefore limited to contexts of especially difficult searches (55). Future studies that examine the effect of search difficulty on distractor inhibition at the neural level are necessary to test the idea that advance distractor suppression may selectively occur in cognitively demanding situations, and whether alpha contributes to this.

\section{Indirect preparatory distractor suppression}

While it is currently unclear whether 'templates for rejection' can be implemented in a voluntary, top-down manner, there is a general consensus that repeated encounters with visual distractors reduces their propensity to capture attention. In the below, we discuss whether such learned inhibition, which does not seem to depend on working memory, is evident in changes in preparatory neural activity (or firing) in regions representing the distractor. First, however, we review recent evidence showing that distractor interference can also be reduced by rendering the representation of the target in working memory maximally distinct from that of the distractor, thereby indirectly decreasing the chance of distractor selection (17).

\section{Indirect distractor suppression through template-to-distractor distinctiveness}

Distractor interference is considerably reduced when observers can define a clear attentional set that is accurately tuned to target-defining features. This is elegantly demonstrated by studies that either encourage searching for a target that is unique in a specific feature dimension, without making the exact feature explicit (e.g., color; singleton-detection mode), and studies that do allow for a precise attentional template (e.g., red; feature-search mode) $(56,57)$. Distractor interference is greatly reduced when attentional (target) templates are feature specific, but not when they are not (56-58). Although challenged by some (59), this dissociation indicates that distractors, even when similar to the target, can be more efficiently ignored when the search allows for goal-directed feature selection. Intriguingly, recent evidence indicates that the (neural) representation of the target template in working memory does not necessarily need to be a veridical copy of the target, but is a highly dynamic representation that can be flexibly adapted to also incorporate distractor 
information (26, 60-62). That is, the target template representation can be strategically shifted 'off-veridical' to optimize the ability to distinguish targets from distractors and thereby improve attentional selectivity (17). Finetuning of the target template in relation to distractor features may take place in lateral prefrontal cortex due to the abundance of neurons with mixed selectivity in this area. A recent study demonstrated that information in working memory in monkey's lateral prefrontal cortex reorganized into a different pattern of activity upon distractor presentation, whereas the same code remained stable in frontal eye fields (63). A challenge for future work will be to establish how such code morphing or template-to-distractor distinctiveness (17) may help, albeit indirectly, the suppression of distractor processing, especially when targets and distractors are highly similar at the feature level. In particular, it is still unclear whether only the target representation is adjusted, or whether the representation of both targets and distractors can be adapted. To summarize, one way to suppress distracting information may be to optimize the distinctiveness of target versus distractor representations, thereby indirectly reducing the ability of distracting information to capture attention.

\section{Expectation suppression}

A form of inhibition that does not seem to rely on representation in working memory and as such may also prevent distractor capture is suppression driven by previous experiences (64) or statistical learning (65). A growing body of work indicates that just like target selection is shaped by stimulus probabilities $(66,67)$, statistical regularities both in the non-spatial $(29,68,69)$ as well as the spatial domain $(16,65,70-73)$ modulate distractor interference. For example, although color singletons often capture attention (74), performance costs become reliable smaller when the color singleton recurs on subsequent searches (69). Similarly, in search tasks, subjects are faster in responding to targets when a distractor is presented more often at one of the search locations, i.e., when its location has become predictable. Crucially, this benefit of distractor predictability cannot be explained by more attention to the remaining possible target locations $(73)$ or mere priming $(75,76)$. Of further note, observers are typically unaware of the unequal probability of distractor occurrences across display locations, indicating that this form of suppression relies on implicit learning mechanisms, i.e., is not dependent on working memory $(16,65)$. In direct support of the notion that statistical distractor learning does not rely on working memory, Gao and Theeuwes (77) recently showed that learning to suppress distracting information was not affected by the load of a concurrent working memory task. 
One recent proposal is that this form of learned inhibition relies on expectation suppression $(9,46)$, consistent with predictive processing models, that have recently gained a lot of scientific traction and stature (78). In these models, the brain continuously generates predictions about incoming sensory input based on learned regularities in the environment, and what is being fed up the hierarchy is not sensory input per se, but rather the mismatch between the brain's a priori predictions and the incoming input, or so-called prediction errors. In this framework, processing of any expected stimulus, whether relevant or irrelevant is thus suppressed (explained 'away'), which should reduce distractor processing and interference. These informed predictions, which need not necessarily be conscious, provide an elegant solution to profit from the abundant statistical regularities that we encounter in our daily environments $(18,19)$. An important outstanding question is how distractor inhibition via expectation suppression is neurally implemented. Specifically, it is unclear whether learned inhibition is implemented already in anticipation of distracting input through modulation of activity in visual regions representing the distracting information, or whether it operates via synaptic plasticity and thus only becomes apparent once distractor knowledge can be integrated with bottom-up sensory input.

The question whether learned inhibition can be preparatory mirrors an ongoing debate in the predictive processing literature, which centers on the question if expectations exert their influence already in advance (79, 80) or alternatively, only become apparent after stimulus presentation $(81,82)$. In line with the notion that the brain continuously generates expectations about upcoming sensory input based on learned regularities in the environment $(79,80)$, expectations have been associated with changes in pre-stimulus sensory activity. For example, a recent MEG study showed expectation-dependent sensory templates already before stimulus onset (83). Expectations (stimulus likelihood) and attention (stimulus relevance) also interact, such that top-down biasing, as reflected in pre-stimulus alpha-lateralization, is most pronounced when targets are also most likely occur at the cued, task-relevant location (84). Thus, expectations may modulate activity in corresponding sensory regions in advance to facilitate goal-directed behavior. Nevertheless, it is still debated how early expectations modulate stimulus processing, with several recent EEG studies suggesting that expectations may primarily affect later stages of information processing $(84,85)$. In addition, how distractor-specific expectations may help resolve interference at the neural level remains unclear, as very few neuroimaging studies have so far investigated how distractor learning helps to filter out visual distractions.

Two recent EEG studies examined whether learning about the likely upcoming location of a distractor stimulus was associated with enhanced pre-stimulus alpha-band activity over contralateral visual regions, and 
provided mixed results. In line with the notion of top-down predictive processing, and the notion of alpha as inhibition, one study demonstrated increased alpha-band activity contralateral to high probability distractor locations already in anticipation of search display onset (86). By contrast, in the study by Noonan et al. (46), as discussed above, alpha-band modulations were only observed in response to target cues, but not in response to distractor cues, not even when the cued distractor location was fixed in a block of trials (see also (76)). One potential explanation for this apparent discrepancy in findings is that in the first study, the shapes and colors of targets and distractors randomly alternated across trials, whereas targets and distractors had fixed identities in the second study. In the second study, distractor learning could thus occur at the feature level, whereas in the first study, it could only occur based on spatial regularities. Alpha-band oscillations may thus specifically implement learning-related space-based suppression. Indeed, recent behavioral studies suggest that the locus of suppression is flexible, and that how distractor location learning changes the representation of distracting information depends on whether the targets and distractors can be identified based on separate features and/or dimensions (e.g., color for distractors and orientation for targets) or not. For example, it has been shown that when the target and distractor cannot be dissociated on the basis of dimension-specific information, target processing is also slowed down at high probably distractor locations $(16,65,87)$, suggesting that inhibition is implemented at a higher-level spatial priority or master saliency map (71). Yet, when the defining dimensions of targets and distractors not only differ, but are also predictable, responses to targets at high probability distractor locations no longer slow down, suggestive of non-spatial or dimension-specific inhibition $(71,75)$. Note however, that typically, in these tasks, both the dimension of the target and the distractor are known in advance, rendering it possible that observed effects simply reflect greater attention to the target dimension rather than inhibition of the distractor dimension per se. Future work is also necessary to establish whether preparatory alpha-band suppression is indeed specific to conditions where the inhibitory set can be implemented at the spatial priority map, and if and how statistical learning about distractor features may affect advance distractor representation.

The studies discussed in the above looked at changes in level of preparatory activity. There are now many demonstrations that the information content of neural activity can be disconnected from the overall amount of neural activity (88), rendering it possible that even in the absence of any changes in the overall level of pre-stimulus activity, distractor expectations change the quality of the sensory representation of the distracting information. We consider three ways in which expectations about upcoming distracting information may change the representation of that information (Figure 1). First, just like expectations about upcoming target 
information may increase the representational content of neural activity, as discussed above, expectations about upcoming distractor information may also result in anticipatory distractor tuning (Figure $1 \mathrm{~A}$ ). If the distractor is already represented in the preparatory neural code, it may subsequently elicit a weaker response (i.e., prediction error), thereby reducing distractor interference. In this scenario, expectations 'silence' information processing regardless of its task relevance. However, in some predictive processing accounts, attention is proposed to regulate the relative influence of prior expectations by controlling the weight of, or precision assigned to, prediction errors (18). In this way, otherwise small prediction errors triggered by expected task-relevant stimuli can be assigned greater value, reversing expectation-dependent suppression (89). A second possibility is thus that distractor learning suppresses anticipatory tuning to expected distractor features by downregulating the weights on corresponding sensory units, resulting in negative anticipatory distractor tuning, effectively 'cancelling' distractor processing (Figure 1B). Note that in this latter scenario it is important to establish in empirical studies whether negative tuning, if observed, is selectively driven by distractor expectations, as a negative tuning slope may also solely arise as a consequence of shifting sensory tuning away from the expected distractor location/features or some combination of both reduced tuning to the expected distractor location/features and enhanced tuning towards less likely distractor locations/features (17) (Figure 1B 1-3). A third, and last possibility, discussed in more detail below, is that distractor expectations are not associated with any changes in anticipatory tuning (Figure 1C) and are only expressed upon distractor presentation.

$\ll<<<<<<$ Insert Figure 1 around here $\gg \gg \gg \gg \gg>>$

In a recent study, we addressed the outstanding question as to whether and how distractor expectations may change the sensory representation of distracting information (76). Specifically, combining EEG and inverted encoding modeling (90), we investigated if distractor location learning, induced by keeping the distractor location stable over trials (i.e., four repetitions), was associated with changes in anticipatory spatial tuning to the distractor location. While distractor location foreknowledge was associated with clear behavioral benefits, it did not result in any changes in preparatory spatial tuning to the distractor location (Figure 2A; bottom row). Note however, that, although highly variable across participants and thus far from statistically robust, there was a hint of negative tuning prior to search display onset. It is possible that the limited number 
of repetitions was only sufficient for a small subset of participants to instantiate negative anticipatory tuning and that with more opportunity to learn, negative distractor tuning may have become reliable. It is also possible that strategies differed across participants. In contrast, and in line with previous studies of spatial attention (9193), repeating the target location was associated with reliable spatial tuning to the target location in advance of stimulus presentation (Figure 2A; top row). Thus, we found a dissociation in that only expectations about upcoming relevant information, not about upcoming irrelevant information, were associated with changes in the representational content of visual activity prior to stimulus presentation. The lack of changes in preparatory tuning to distractor features may support the notion that distractor expectations exert their effects only once confronted with the distracting information (Figure 1C). It is notable in this respect, as described in more detail below, that distractor learning was associated with changes in post-distractor processing.

Based on the evidence reviewed above, we conclude that there is currently very little evidence in support of preparatory inhibition, as indexed by changes in the pattern of activity in visual regions representing the distracting information in anticipation of upcoming distracting input, even after statistical learning. As discussed below, however, this does not necessarily imply that distractors always capture attention, as growing evidence indicates that distractors can be filtered out pre-attentively.

\footnotetext{
$\ll<<<<<<\mid$ Insert Figure 2 around here $\gg \gg \gg \gg \gg>>>$
}

\section{Post-distractor inhibition: Pre-attentive and reactive inhibition}

A parallel, but largely separate debate in the literature, next to the question as to whether it is possible to prepare the system in advance to suppress sensory processing of distracting information and whether this relies on representation in working memory, has concerned the question whether it is possible to suppress distractors before they capture attention. This debate has primarily focused on suppression of physically salient distractors (e.g., a uniquely colored object amongst homogenously colored search items) by examining whether they can be suppressed before they capture attention (i.e. pre-attentively) $(10,94)$, or only reactively, after attentional selection $(7,13)$.

At first sight, distractor suppression in the absence of attentional selection may appear paradoxical as one would assume that to filter out a specific item, that item must first, at least to some degree, be selected. 
This ignoring paradox is evident in the 'search and destroy' hypothesis advocated by Moher and Egeth (13), which argues that distractors automatically capture attention and then only subsequently (i.e., reactively) are suppressed. Recently, however, it was postulated that even salient distractors that generate a strong bottomup saliency signal can be suppressed before they capture attention, pre-attentively $(10,94)$. Although in the original conception of this so-called signal-suppression hypothesis, it was assumed that the inhibitory processes are implemented by direct top-down control, more recent conceptions also consider a role for statistical learning as discussed above $(10,94)$. To dissociate between these competing hypotheses, behavioral studies have used capture probe paradigms, where probe displays are randomly intermixed with search displays. A lower probability of reporting probes embedded within singleton distractors has been taken as evidence in support of the signal suppression hypothesis $(95,96)$. Yet, the same pattern of behavioral results can be explained by reactive suppression in the sense that attention is first captured by the singleton distractor (if only for the briefest moment) and subsequently immediately suppressed (97).

To bypass the ambiguities of behavioral evidence, researchers have turned to two event-related potentials in the electrophysiological (EEG) signal: the N2pc and the Pd. These typically occur around $200 \mathrm{~ms}$ post-stimulus onset, and reflect attentional selection (98-100) and suppression $(95,101)$, respectively. The Pd, a transient positivity contralateral to the distractor, is especially relevant as its amplitude inversely scales with behavioral measures of distractor interference (102-104). It is selectively elicited by distractors, and is independent from other lateralized components reflective of attentional selection, such as the $\mathrm{N} 2 \mathrm{pc}(76,94$, $101,105)$ and N1pc (105). Accordingly, the Pd has been proposed to reflect a mechanism that prevents or terminates the allocation of attention towards a salient distractor $(10,106)$.

Although in many cases the Pd follows the N2pc $(76,103,107-109)$ or N1pc $(105)$ in the ERP waveform as predicted by the ignoring paradox, growing evidence indicates that salient distractors can also be inhibited (as evidenced by a Pd) in the absence of any neural evidence for attentional selection (e.g., the absence of an N2pc) (10). Notably, in the majority of these studies, the experimental design allowed for statistical learning, either because the target and distractor identities were fixed (i.e., same color/shape) across trials $(95,102,110$, 111), or because there was a high probability distractor location (86). This suggest that experience with distracting information is necessary for pre-attentive suppression of distracting information. However, salient distractors have also been found to elicit a Pd in the absence of an N2pc in ERP studies with search display 
configurations that did not allow for the formation of distractor-specific expectations (e.g., when the location and color of the target and singleton distractor vary from trial to trial) (94), or when search displays were not presented until response, but only briefly (107), and when analysis were limited to fast trials only (112). These findings are difficult to reconcile with the notion of expectation-dependent distractor suppression, and suggest that salient distractors can also be filtered out pre-attentively in other ways. Indeed, distractor inhibition may also be based on feature discontinuity $(23,113,114)$ or global, dimension-independent salience $(10,115)$. Note that such feature-unspecific distractor inhibition should also not be associated with a precise distractor template in working memory, which could thereby reduce the chance of attentional capture. An important question for future research is how distractor feature-specific and unspecific inhibition are differentially implemented at the neural level, and the extent to which they are dependent on statistical learning.

It is typically assumed that pre-attentive suppression relies on proactive mechanisms set up before stimulus presentation (116). However, as discussed above there is currently little evidence in support of preparatory distractor-specific sensory inhibition at the neural level. In this respect it is important to note that the majority of studies examining pre-attentive suppression are agnostic about the underlying neural mechanisms as they only focused on distractor-evoked responses such as the Pd. Rather than modulating activity in regions representing the distracting information, however, distractor learning could also change synaptic efficiency within these regions, analogous to long-term visual recognition memory (117) and activitysilent coding in working memory (118). Synaptic memory traces provide a more efficient coding scheme than active suppression through inhibition, and could explain longer lasting effects of learning on distractor interference. The notion of synaptic plasticity as a mechanism underlying statistical learning can also be reconciled with proposals that expectations exert their influence only after the bottom-up stimulus has been initially processed, during later stages of sensory processing $(84,119)$.

Recent non-human primate work suggests that the frontal eye fields (FEF) and lateral intraparietal (LIP) cortex play an important role in integrating top-down expectations with bottom-up input in feature-specific inhibition (120-122). While typically responses in these areas increase as a function of saliency $(123,124)$, as the animals learn to ignore salient distractors, evoked responses to those distractors become smaller than responses elicited by non-salient distractors. In a study by Cosman and colleagues (120), the suppressed FEF response, which was observed once the learned to-be-ignored distractor no longer incurred a behavioral cost, was furthermore followed by a scalp-recorded Pd-like component, suggesting that the frontal eye fields play an 
important role in implementing inhibition. In combination with previous work showing that V4 responses initially do not differentiate between targets and distractors (125), these findings support the notion that distractor inhibition can be instantiated after distractor learning, once the to-be-suppressed stimulus is physically presented and bottom-up information can be integrated with top-down influences. The FEF and LIP may play a critical role in this.

Notably, several recent studies in humans have associated distractor learning with decreases in the amplitude of the Pd ERP component, indicative of a reduced distractor inhibition $(76,126,127)$. In our recent study (76), for example, the Pd was virtually eliminated when the location of the distractor was learned, in the absence of any changes in early visual processing, as reflected in the amplitude of the early visual-evoked P1 and N1 ERP components, or any modulations of the N2pc (Figure 2C). This finding may suggest that the brain no longer considered distractors as distractors, once it had learned that they could be safely ignored. As noted above and shown in Figure $2 \mathrm{~A}$, in our study we also observed no anticipatory spatial tuning towards expected distractor locations. These findings may thus provide further support for the idea that distractor learning is expressed post-distractor through integration of top-down influences and bottom-up information, just like target learning (119) possibly through changes in synaptic efficiency (118).

In sum, the evidence reviewed above indicates that although suppression of physically salient distractors often occurs reactively, other mechanisms may intervene to filter out salient distractors preattentively, such that capture is entirely prevented. Such inhibition of physically salient distractors on the basis of individual visual features in particular seems dependent on prior experience with the distracting information, to prevent working memory template-driven attentional capture. Future research will need to more precisely establish how such distractor inhibition is neurally implemented and to what extent this depends on the level at which suppression operates: based on spatial information and/or features, feature discontinuity (23) or global, dimension-independent salience (115). Few studies have also so far investigated if other types of physically salient stimuli (e.g., sudden onsets) can be suppressed. Recent behavioral work suggests that distraction by sudden onsets can be eliminated through mere passive viewing (128), indicative of latent learning mechanisms or habituation. How learning through passive viewing may be related to statistical learning/predictive processing is another important avenue for future research (6). Finally, information about upcoming distraction can also be non-specific, but nevertheless used to prepare the system more globally, for example, through global suppression of sensory processing. 


\section{Inhibition in task-relevant networks}

So far, we have discussed neural mechanisms underlying inhibition of distracting information, and pointed out the relative lack of support for the notion that alpha oscillations implement direct top-down inhibition (Forster \& Awh, 2019). This is not to say that alpha oscillations do not functionally reflect inhibition. Notably, a growing body of work suggests that alpha oscillations play an important role in biasing visual regions towards processing of task-relevant information. That is, while the evidence for alpha-band oscillatory activity in direct, top-down inhibition is ambiguous, as discussed above, many studies have now shown that the focus of attention can be decoded from the pattern of alpha-band EEG activity even before stimulus presentation (91-93). These and other findings support the notion that one important role for alpha oscillations may be to enhance signal-to-noise ratio within task-relevant regions by suppressing noise (20). Suppression of neuronal activity within taskrelevant sensory regions through alpha-band oscillations could, when at an intermediate level, suppress activity of neurons with low activity to begin with, but not of neurons with high activity to begin with (i.e., those representing the attended, task-relevant information), thereby increasing the signal-to-noise ratio. Rather than playing a role in inhibition per se, alpha oscillations may thus enhance tuning to task-relevant features in visual regions by selectively suppressing activity of neurons tuned to other features. Interestingly, and furthermore in line with this possibility, recent studies have related increased alpha-band activity to more stable visual percepts, leading to the proposal that alpha oscillations may not signal inhibition of cortical activity per se, but stabilization of the current configuration of neuronal activity $(21,22)$. From this perspective, the observed distractor location learning-related increase in pre-stimulus alpha activity in the study by Wang and colleagues (86) discussed above, could also denote sharper representation of distracting information (Figure 1A), not preparatory inhibition of expected distractor information. This is a radically different interpretation, and more work is necessary to establish to what extent alpha-band oscillations implement preparatory inhibition of distracting information (cf. Figure 1B) or allow for a more precise representation of the distracting information (cf. Figure 1A).

Next to enhancing signal-to-noise, alpha oscillations may also facilitate processing of task-relevant information by creating periods of optimal information processing or 'pulsed' inhibition (129). It has been proposed that oscillatory alpha activity operates in a phasic manner, alternating between phases of relatively greater inhibition and relatively reduced inhibition/greater excitability $(130,131)$. Indeed, it has been shown 
that at certain phases of the alpha cycle visual-evoked ERPs are larger and stimulus detection ability is higher than at other phases $(132,133)$. Yet, it is still unclear whether this alignment in time of the most optimal alpha phase with incoming input is under voluntary control (134). Moreover, this mechanism is only useful in situations in which the timing of goal-relevant information can be predicted with relatively high precision (135). Furthermore, alpha phase may only modulate processing of at-threshold visual stimuli, not of clearly visible stimuli that evoke a strong bottom-up response (136). Finally, pre-stimulus alpha phase does not influence auditory processing and detection (137). Thus, pulsed inhibition through alpha phase may be specific to the visual domain and only of value in very specific conditions. An interesting question for future research is nevertheless whether alpha phase can also be adjusted when the timing of distracting information is highly predictable.

To summarize, inhibition implemented by alpha-band oscillations may play a critical role in taskrelevant sensory networks by suppression noise and creating optimal time windows for information processing through pulsed inhibition. An important question is whether alpha oscillations play a similar role within taskirrelevant visual networks. Specifically, future research should clarify whether alpha-band oscillations may reduce distractor interference by allowing the brain to more precisely represent the distracting information in the same way as they may sharpen target representations, or through preparatory inhibition of distractor representations.

\section{Conclusions and future directions}

Until very recently it was typically assumed in the cognitive neuroscience literature that inhibition in selective attention is under similar flexible control as the selection of relevant information $(4,138)$. The evidence reviewed here, however, demonstrates that the neural mechanisms underlying distractor inhibition differ, at least to a large extent, from the ones that guide attention in space or along other feature dimensions. While attention can flexibly bias visual regions in advance to boost processing of goal-relevant information $(139,140)$, distractor foreknowledge often hampers performance, unless a defining distractor property (e.g., it's location or color) becomes predictable through statistical learning $(6,9,10,65)$. Yet, how such learned inhibition is neurally implemented remains an important outstanding question for future research. Based on notions of predictive processing $(18,19)$, we outlined several ways in which expectations about upcoming distracting information 
grounded in statistical learning may modulate the sensory representation of distracting information and distractor processing (Figure 1), which require further investigation. While there is now abundant evidence that salient distractors can be filtered out pre-attentively (10), the majority of these studies did not examine potential changes in anticipatory activity. It therefore remains unclear whether learned inhibition modulates activity in regions representing the distracting information in advance, or alternatively, exerts its effect in interaction with the bottom-up input, for example, through synaptic plasticity.

The few studies that did examine effects of distractor learning on pre-stimulus activity only manipulated spatial probability and provided mixed results, with one study reporting a role for pre-stimulus alpha-band activity (86), whereas another study did not (46). This apparent discrepancy can be explained by assuming a flexible locus of distractor suppression, which either in case of only spatial regularities operates at the level of spatial priority maps, or in case of additional feature expectations operates at so called conspicuity maps coding specific feature dimensions (141). Given the tight link between alpha-band activity and spatial attention (142), suppression operating at spatial priority maps likely relies on modulations of alpha oscillations. More work, however, is necessary to determine the functional significance of alpha-band oscillatory activity, especially since recent work indicates that alpha oscillations may not signal top-down inhibition of cortical activity per se, but stabilization of the current configuration of neuronal activity $(21,22)$, possibly through enhancing signal-to-noise (20). Distractor expectation-dependent increases in pre-stimulus alpha activity thus may not necessarily reflect preparatory inhibition (Figure 1B), but could also denote a more precise anticipatory representation of distracting information (Figure 1A), which has very different theoretical implications. With the recent advance of new encoding techniques that use the topographic distribution of M/EEG signals to track how spatial attention is deployed via so called spatial tuning functions $(91,93)$, we can now address this outstanding issue. In a first study, however, we did not observe any learning-related changes in anticipatory tuning to an expected distractor location (59) (Figure 2A). Encoding techniques also allow for reconstructions of non-spatial information (143), so that future work can establish whether feature-based suppression can (also) be localized within anticipatory activation patterns, and how spatial and feature distractor foreknowledge may interact to reduce distractor interference. This work will be essential for furthering our understanding as to how expectations may be differentially implemented depending on the relevance or irrelevance of information.

Further studies are also necessary to gain a better understanding of the brain regions and networks that play a key role in distractor learning and statistical learning, more generally. Distractor learning likely 
depends on the hippocampus and subcortical regions, specifically the basal ganglia and thalamus. The hippocampus records the relations between aspects of an experience, such as its sensory components encoded by the neocortex, by providing a spatial and temporal context, and stores this representation of the experience into long-term memory (144-148). Notably, visual and memory systems are reciprocally connected (149) raising the possibility that the hippocampus is the source of expectation-based influences on visual processing. Indeed, recent $\mathrm{fMRI}$ work shows that representations in the hippocampus code predicted shapes (150). The basal ganglia (151) and pulvinar (152) have also been associated with implicit learning, predictive processing, and distractor filtering (153). Lastly, effects of distractor learning may be expressed in priority maps in frontal and parietal cortex $(16,154)$, as also suggested by studies in non-human primates (120-122). Plastic changes in priority maps of space in fronto-parietal cortex can account for the fact that attentional priority at a given location is increased or decreased depending on whether that location is associated with a target or a distractor, and observations from behavioral studies that also processing of targets presented at a likely distractor location is impaired (16, $65,71)$, at least when targets and distractors cannot be distinguished at the dimension level (75). How cortical and subcortical regions interact to implement learned inhibition is another important outstanding question for future studies.

That distractor filtering capitalizes on experience with the distractor information agrees with characteristics of habituation, the progressive attenuation of the amplitude of responses to repeated sensory stimulation which is not caused by sensory adaptation or motor fatigue (155). Although, thus far, habituation has especially been shown to account for reduced distractor interference following repeated exposure to visual onset distractors (156-158), it's defining characteristics resonate with the idea that only prediction errors capture attention (6). Just as in predictive coding models where the brain is continuously trying to predict new sensory input based on previous experiences, in habituation models, sensory input is also compared against stored representations based on expected frequency and the context (159). Intriguingly, a habituated response to visual onsets can even be instantiated during passive viewing (128), indicating that distractor filtering can emerge independent from any attentional biases implemented by task set. It is well established that habituation effects disappear spontaneously over time when the inducing stimulus is withheld (160). Indeed, a recent study reported that in case of short-term habituation (across 100 trials/16min), $16 \mathrm{~min}$ of distractor removal were sufficient to observe a recovery of attentional capture by the distractor (157). Yet, it was also shown that effects of longer experience with the distractors were still visible 24 and 48 hours later, in line with the notion that 
distractor learning can have post-synaptic effects that have long-term effects on attentional selection (161). An important avenue for future research is to determine effects of much longer distractor learning, e.g., across multiple days or weeks, on distractor processing at the neural level.

To conclude, selective attention critically relies on the ability to suppress distracting information. The evidence reviewed here suggests that the brain can proactively inhibit distracting information through integration of bottom-up input and top-down influences, but this ability appears to strongly depend on previous experience with the distracting information, at least when implemented at the feature level. Additional research is necessary to establish how distractor learning affects distractor processing, through preparatory suppression of activity in regions representing the distractor or synaptic plasticity, and to identify the specific brain networks involved.

\section{Competing interest}

The authors declare no competing financial interests or any other conflict of interest 


\section{References}

1. Desimone R, Duncan J. Neural mechanisms of selective visual attention. Annual review of neuroscience. 1995;18(1):193-222.

2. Buschman TJ, Kastner S. From behavior to neural dynamics: an integrated theory of attention. Neuron. 2015;88(1):127-44.

3. Reynolds JH, Heeger DJ. The normalization model of attention. Neuron. 2009;61(2):168-85.

4. Jensen $\mathrm{O}$, Mazaheri A. Shaping functional architecture by oscillatory alpha activity: gating by inhibition. Frontiers in human neuroscience. 2010;4:186.

5. Gazzaley A, Nobre AC. Top-down modulation: bridging selective attention and working memory. Trends in cognitive sciences. 2012;16(2):129-35.

6. Chelazzi L, Marini F, Pascucci D, Turatto M. Getting rid of visual distractors: The why, when, how and where. Current opinion in psychology. 2019.

7. Geng JJ. Attentional mechanisms of distractor suppression. Current Directions in Psychological Science. 2014;23(2):147-53.

8. Kastner $S$, Ungerleider LG. The neural basis of biased competition in human visual cortex. Neuropsychologia. 2001;39(12):1263-76.

9. Noonan MP, Crittenden BM, Jensen O, Stokes MG. Selective inhibition of distracting input. Behavioural brain research. 2018;355:36-47.

10. Gaspelin N, Luck SJ. The role of inhibition in avoiding distraction by salient stimuli. Trends in cognitive sciences. 2018;22(1):79-92.

11. Arita JT, Carlisle NB, Woodman GF. Templates for rejection: configuring attention to ignore task-irrelevant features. Journal of experimental psychology: human perception and performance. 2012;38(3):580.

12. Woodman GF, Luck SJ. Do the contents of visual working memory automatically influence attentional selection during visual search? Journal of Experimental Psychology: Human Perception and Performance. 2007;33(2):363.

13. Moher J, Egeth HE. The ignoring paradox: Cueing distractor features leads first to selection, then to inhibition of to-be-ignored items. Attention, Perception, \& Psychophysics. 2012;74(8):1590-605.

14. Geng JJ, Won B-Y, Carlisle NB. Distractor ignoring: strategies, learning, and passive filtering. Current Directions in Psychological Science. 2019:0963721419867099.

15. Wang B, Theeuwes J. How to inhibit a distractor location? Statistical learning versus active, top-down suppression. Attention, Perception, \& Psychophysics. 2018:1-11.

16. Ferrante O, Patacca A, Di Caro V, Della Libera C, Santandrea E, Chelazzi L. Altering spatial priority maps via statistical learning of target selection and distractor filtering. Cortex. 2018;102:67-95.

17. Geng JJ, Witkowski P. Template-to-distractor distinctiveness regulates visual search efficiency. Current opinion in psychology. 2019.

18. Friston $\mathrm{K}$. The free-energy principle: a rough guide to the brain? Trends in cognitive sciences. 2009;13(7):293-301.

19. Rao RP. Bayesian inference and attentional modulation in the visual cortex.

Neuroreport. 2005;16(16):1843-8.

20. Klimesch W. Alpha-band oscillations, attention, and controlled access to stored information. Trends in cognitive sciences. 2012;16(12):606-17. 
21. Clayton MS, Yeung N, Cohen Kadosh R. Electrical stimulation of alpha oscillations stabilizes performance on visual attention tasks. Journal of Experimental Psychology: General. 2019;148(2):203.

22. Piantoni G, Romeijn N, Gomez-Herrero G, Van Der Werf YD, Van Someren EJ. Alpha power predicts persistence of bistable perception. Scientific reports. 2017;7(1):5208.

23. Wolfe JM. Guided search 2.0 a revised model of visual search. Psychonomic bulletin \& review. 1994;1(2):202-38.

24. Olivers CN, Peters J, Houtkamp R, Roelfsema PR. Different states in visual working memory: When it guides attention and when it does not. Trends in cognitive sciences. 2011;15(7):327-34.

25. van Moorselaar D, Theeuwes J, Olivers CN. In competition for the attentional template: Can multiple items within visual working memory guide attention? Journal of Experimental Psychology: Human Perception and Performance. 2014;40(4):1450.

26. Navalpakkam V, Itti L. Search goal tunes visual features optimally. Neuron. 2007;53(4):605-17.

27. Becker MW, Hemsteger S, Peltier $C$. No templates for rejection: A failure to configure attention to ignore task-irrelevant features. Visual Cognition. 2015;23(9-10):1150-67.

28. Beck VM, Hollingworth A. Evidence for negative feature guidance in visual search is explained by spatial recoding. Journal of Experimental Psychology: Human Perception and Performance. 2015;41(5):1190.

29. Cunningham CA, Egeth HE. Taming the white bear: Initial costs and eventual benefits of distractor inhibition. Psychological science. 2016;27(4):476-85.

30. Park S, Kim M-S, Chun MM. Concurrent working memory load can facilitate selective attention: evidence for specialized load. Journal of Experimental Psychology: Human Perception and Performance. 2007;33(5):1062.

31. Carlisle NB, Nitka AW. Location-based explanations do not account for active attentional suppression. Visual Cognition. 2018:1-12.

32. Munneke J, Van der Stigchel S, Theeuwes J. Cueing the location of a distractor: An inhibitory mechanism of spatial attention? Acta Psychologica. 2008;129(1):101-7.

33. Chao H-F. Top-down attentional control for distractor locations: The benefit of precuing distractor locations on target localization and discrimination. Journal of Experimental Psychology: Human Perception and Performance. 2010;36(2):303.

34. Foxe JJ, Snyder AC. The role of alpha-band brain oscillations as a sensory suppression mechanism during selective attention. Frontiers in Psychology. 2011;2:154.

35. Haegens $S$, Nácher V, Luna R, Romo R, Jensen O. $\alpha$-Oscillations in the monkey sensorimotor network influence discrimination performance by rhythmical inhibition of neuronal spiking. Proceedings of the National Academy of Sciences. 2011;108(48):19377-82.

36. Worden MS, Foxe JJ, Wang N, Simpson GV. Anticipatory biasing of visuospatial attention indexed by retinotopically specific-band electroencephalography increases over occipital cortex. J Neurosci. 2000;20(RC63):1-6.

37. Thut G, Nietzel A, Brandt SA, Pascual-Leone A. $\alpha$-Band electroencephalographic activity over occipital cortex indexes visuospatial attention bias and predicts visual target detection. Journal of Neuroscience. 2006;26(37):9494-502.

38. Kelly SP, Lalor EC, Reilly RB, Foxe JJ. Increases in alpha oscillatory power reflect an active retinotopic mechanism for distracter suppression during sustained visuospatial attention. Journal of neurophysiology. 2006;95(6):3844-51. 
39. Rihs TA, Michel CM, Thut G. Mechanisms of selective inhibition in visual spatial attention are indexed by $\alpha$-band EEG synchronization. European Journal of Neuroscience. 2007;25(2):603-10.

40. Snyder AC, Foxe JJ. Anticipatory attentional suppression of visual features indexed by oscillatory alpha-band power increases: a high-density electrical mapping study. Journal of Neuroscience. 2010;30(11):4024-32.

41. Jokisch $\mathrm{D}$, Jensen $\mathrm{O}$. Modulation of gamma and alpha activity during a working memory task engaging the dorsal or ventral stream. Journal of Neuroscience. 2007;27(12):3244-51.

42. Romei V, Thut G, Mok RM, Schyns PG, Driver J. Causal implication by rhythmic transcranial magnetic stimulation of alpha frequency in feature-based local vs. global attention. European Journal of Neuroscience. 2012;35(6):968-74.

43. Foster JJ, Awh E. The role of alpha oscillations in spatial attention: Limited evidence for a suppression account. Current opinion in psychology. 2018.

44. Munneke J, Heslenfeld DJ, Usrey WM, Theeuwes J, Mangun GR. Preparatory effects of distractor suppression: Evidence from visual cortex. PloS one. 2011;6(12):e27700.

45. Couperus J, Mangun GR. Signal enhancement and suppression during visual-spatial selective attention. Brain research. 2010;1359:155-77.

46. Noonan MP, Adamian N, Pike A, Printzlau F, Crittenden BM, Stokes MG. Distinct mechanisms for distractor suppression and target facilitation. Journal of Neuroscience. 2016;36(6):1797-807.

47. de Vries IE, Savran E, van Driel J, Olivers CN. Oscillatory mechanisms of preparing for visual distraction. Journal of cognitive neuroscience. 2019:1-22.

48. Bonnefond $\mathrm{M}$, Jensen $\mathrm{O}$. Alpha oscillations serve to protect working memory maintenance against anticipated distracters. Current biology. 2012;22(20):1969-74.

49. Reeder RR, Olivers CN, Pollmann S. Cortical evidence for negative search templates. Visual Cognition. 2017;25(1-3):278-90.

50. Reeder RR, Olivers CN, Hanke M, Pollmann S. No evidence for enhanced distractor template representation in early visual cortex. Cortex; a journal devoted to the study of the nervous system and behavior. 2018;108:279-82.

51. Awh E, Matsukura M, Serences JT. Top-down control over biased competition during covert spatial orienting. Journal of Experimental Psychology: Human Perception and Performance. 2003;29(1):52.

52. Serences JT, Yantis S, Culberson A, Awh E. Preparatory activity in visual cortex indexes distractor suppression during covert spatial orienting. Journal of Neurophysiology. 2004.

53. Ruff CC, Driver J. Attentional preparation for a lateralized visual distractor:

Behavioral and fMRI evidence. Journal of Cognitive Neuroscience. 2006;18(4):522-38.

54. Marini F, Demeter E, Roberts KC, Chelazzi L, Woldorff MG. Orchestrating proactive and reactive mechanisms for filtering distracting information: Brain-behavior relationships revealed by a mixed-design fMRI study. Journal of Neuroscience. 2016;36(3):988-1000.

55. Conci M, Deichsel C, Müller HJ, Töllner T. Feature guidance by negative attentional templates depends on search difficulty. Visual Cognition. 2019:1-10.

56. Bacon WF, Egeth HE. Overriding stimulus-driven attentional capture. Perception \& psychophysics. 1994;55(5):485-96.

57. Leber $A B$, Egeth HE. It's under control: Top-down search strategies can override attentional capture. Psychonomic bulletin \& review. 2006;13(1):132-8. 
58. Lamy D, Leber A, Egeth HE. Effects of task relevance and stimulus-driven salience in feature-search mode. Journal of Experimental Psychology: Human Perception and Performance. 2004;30(6):1019.

59. Theeuwes J. Top-down search strategies cannot override attentional capture. Psychonomic bulletin \& review. 2004;11(1):65-70.

60. Geng JJ, DiQuattro NE, Helm J. Distractor probability changes the shape of the attentional template. Journal of experimental psychology: human perception and performance. 2017;43(12):1993.

61. Yu X, Geng JJ. The attentional template is shifted and asymmetrically sharpened by distractor context. Journal of experimental psychology: human perception and performance. 2019;45(3):336.

62. Becker $\mathrm{SI}$, Folk $\mathrm{CL}$, Remington RW. The role of relational information in contingent capture. Journal of Experimental Psychology: Human Perception and Performance. 2010;36(6):1460.

63. Parthasarathy A, Herikstad R, Bong JH, Medina FS, Libedinsky C, Yen S-C. Mixed selectivity morphs population codes in prefrontal cortex. Nature neuroscience. 2017;20(12):1770-9.

64. Gao Y, Theeuwes J. Learning to suppress a distractor is not affected by working memory load. Psychonomic Bulletin \& Review. 2019.

65. Wang B, Theeuwes J. Statistical regularities modulate attentional capture. Journal of Experimental Psychology: Human Perception and Performance. 2018;44(1):13.

66. Geng JJ, Behrmann M. Spatial probability as an attentional cue in visual search. Perception \& psychophysics. 2005;67(7):1252-68.

67. Jiang YV, Swallow KM, Rosenbaum GM, Herzig C. Rapid acquisition but slow extinction of an attentional bias in space. Journal of Experimental Psychology: Human Perception and Performance. 2013;39(1):87.

68. Stilwell BT, Bahle B, Vecera SP. Feature-based statistical regularities of distractors modulate attentional capture. Journal of experimental psychology: human perception and performance. 2019;45(3):419.

69. Vatterott DB, Vecera SP. Experience-dependent attentional tuning of distractor rejection. Psychonomic bulletin \& review. 2012;19(5):871-8.

70. Goschy H, Bakos S, Müller HJ, Zehetleitner M. Probability cueing of distractor locations: both intertrial facilitation and statistical learning mediate interference reduction. Frontiers in psychology. 2014;5:1195.

71. Sauter M, Liesefeld HR, Zehetleitner M, Müller HJ. Region-based shielding of visual search from salient distractors: Target detection is impaired with same-but not differentdimension distractors. Attention, Perception, \& Psychophysics. 2018;80(3):622-42.

72. Reder LM, Weber K, Shang J, Vanyukov PM. The adaptive character of the attentional system: statistical sensitivity in a target localization task. Journal of Experimental Psychology: Human Perception and Performance. 2003;29(3):631.

73. Failing $M$, Wang $B$, Theeuwes J. Spatial suppression due to statistical regularities is driven by distractor suppression not by target activation. Attention, Perception, \& Psychophysics. 2019:1-10.

74. Theeuwes J. Perceptual selectivity for color and form. Perception \& psychophysics. 1992;51(6):599-606. 
75. Zhang B, Allenmark F, Liesefeld HR, Shi Z, Muller H. Probability cueing of singletondistractor locations in visual search: priority-map-or dimension-based inhibition? Journal of Experimental Psychology: Human Perception and Performance. 2019.

76. van Moorselaar D, Slagter HA. Learning what is irrelevant or relevant: Expectations facilitate distractor inhibition and target facilitation through distinct neural mechanisms. bioRxiv. 2019.

77. Gao Y, Theeuwes J. Learning to suppress a distractor is not affected by working memory load. Psychonomic Bulletin \& Review. 2019:1-9.

78. Friston K. A theory of cortical responses. Philosophical transactions of the Royal Society B: Biological sciences. 2005;360(1456):815-36.

79. Fiser A, Mahringer D, Oyibo HK, Petersen AV, Leinweber M, Keller GB. Experiencedependent spatial expectations in mouse visual cortex. Nature neuroscience. 2016;19(12):1658.

80. Bell AH, Summerfield C, Morin EL, Malecek NJ, Ungerleider LG. Encoding of stimulus probability in macaque inferior temporal cortex. Current Biology. 2016;26(17):2280-90.

81. Bar M, Kassam KS, Ghuman AS, Boshyan J, Schmid AM, Dale AM, et al. Top-down facilitation of visual recognition. Proceedings of the national academy of sciences. 2006;103(2):449-54.

82. Rao RP, Ballard DH. Predictive coding in the visual cortex: a functional interpretation of some extra-classical receptive-field effects. Nature neuroscience. 1999;2(1):79.

83. Kok P, Mostert P, De Lange FP. Prior expectations induce prestimulus sensory templates. Proceedings of the National Academy of Sciences. 2017:201705652.

84. Alilović J, Timmermans B, Reteig LC, Van Gaal S, Slagter HA. No evidence that predictions and attention modulate the first feedforward sweep of cortical information processing. Cerebral Cortex. 2019;29(5):2261-78.

85. Rungratsameetaweemana N, Itthipuripat S, Salazar A, Serences JT. Expectations do not alter early sensory processing during perceptual decision making. Journal of Neuroscience. 2018:3638-17.

86. Wang B, van Driel J, Ort E, Theeuwes J. Anticipatory distractor suppression elicited by statistical regularities in visual search. Journal of cognitive neuroscience. 2019:1-14.

87. Failing M, Feldmann-Wustefeld T, Wang B, Christian O, Theeuwes J. Statistical regularities induce spatial as well as feature-specific suppression. Journal of experimental psychology: human perception and performance. 2019.

88. Kok P, Jehee JF, De Lange FP. Less is more: expectation sharpens representations in the primary visual cortex. Neuron. 2012;75(2):265-70.

89. Feldman $\mathrm{H}$, Friston $\mathrm{K}$. Attention, uncertainty, and free-energy. Frontiers in human neuroscience. 2010;4:215.

90. Foster JJ, Sutterer DW, Serences JT, Vogel EK, Awh E. The topography of alpha-band activity tracks the content of spatial working memory. Journal of neurophysiology. 2015;115(1):168-77.

91. Samaha J, Sprague TC, Postle BR. Decoding and reconstructing the focus of spatial attention from the topography of alpha-band oscillations. Journal of cognitive neuroscience. 2016;28(8):1090-7.

92. van Moorselaar D, Foster JJ, Sutterer DW, Theeuwes J, Olivers CN, Awh E. Spatially selective alpha oscillations reveal moment-by-moment trade-offs between working memory and attention. Journal of cognitive neuroscience. 2018;30(2):256-66. 
93. Foster JJ, Sutterer DW, Serences JT, Vogel EK, Awh E. Alpha-band oscillations enable spatially and temporally resolved tracking of covert spatial attention. Psychological science. 2017;28(7):929-41.

94. Sawaki R, Luck SJ. Capture versus suppression of attention by salient singletons: Electrophysiological evidence for an automatic attend-to-me signal. Attention, Perception, \& Psychophysics. 2010;72(6):1455-70.

95. Gaspelin N, Luck SJ. Combined Electrophysiological and Behavioral Evidence for the Suppression of Salient Distractors. Journal of cognitive neuroscience. 2018:1-16.

96. Won B-Y, Kosoyan M, Geng JJ. Evidence for second-order singleton suppression based on probabilistic expectations. Journal of Experimental Psychology: Human Perception and Performance. 2019;45(1):125.

97. Chang S, Cunningham CA, Egeth HE. The power of negative thinking: Paradoxical but effective ignoring of salient-but-irrelevant stimuli with a spatial cue. Visual Cognition. 2018:1-15.

98. Luck SJ. Electrophysiological correlates of the focusing of attention within complex visual scenes: N2pc and related ERP components. The Oxford handbook of event-related potential components. 2012:329-60.

99. Eimer M. The neural basis of attentional control in visual search. Trends in Cognitive Sciences. 2014;18(10):526-35.

100. Luck SJ, Hillyard SA. Electrophysiological correlates of feature analysis during visual search. Psychophysiology. 1994;31(3):291-308.

101. Hickey C, Di Lollo V, McDonald JJ. Electrophysiological indices of target and distractor processing in visual search. Journal of cognitive neuroscience. 2009;21(4):760-75.

102. Gaspar JM, McDonald JJ. Suppression of salient objects prevents distraction in visual search. Journal of neuroscience. 2014;34(16):5658-66.

103. Liesefeld HR, Liesefeld AM, Töllner T, Müller HJ. Attentional capture in visual search: Capture and post-capture dynamics revealed by EEG. Neurolmage. 2017;156:166-73.

104. Weaver MD, van Zoest W, Hickey C. A temporal dependency account of attentional inhibition in oculomotor control. Neurolmage. 2017;147:880-94.

105. Donohue SE, Bartsch MV, Heinze H-J, Schoenfeld MA, Hopf J-M. Cortical mechanisms of prioritizing selection for rejection in visual search. Journal of neuroscience.

2018;38(20):4738-48.

106. Sawaki R, Geng JJ, Luck SJ. A common neural mechanism for preventing and terminating the allocation of attention. Journal of Neuroscience. 2012;32(31):10725-36.

107. Kiss M, Grubert A, Petersen A, Eimer M. Attentional capture by salient distractors during visual search is determined by temporal task demands. Journal of cognitive neuroscience. 2012;24(3):749-59.

108. Hilimire MR, Corballis PM. Event-related potentials reveal the effect of prior knowledge on competition for representation and attentional capture. Psychophysiology. 2014;51(1):22-35.

109. Sawaki R, Luck SJ. Active suppression after involuntary capture of attention. Psychonomic bulletin \& review. 2013;20(2):296-301.

110. Jannati A, Gaspar JM, McDonald JJ. Tracking target and distractor processing in fixedfeature visual search: evidence from human electrophysiology. Journal of Experimental Psychology: Human Perception and Performance. 2013;39(6):1713. 
111. Burra N, Kerzel D. Attentional capture during visual search is attenuated by target predictability: evidence from the N2pc, Pd, and topographic segmentation.

Psychophysiology. 2013;50(5):422-30.

112. McDonald JJ, Green JJ, Jannati A, Di Lollo V. On the electrophysiological evidence for the capture of visual attention. Journal of Experimental Psychology: Human Perception and Performance. 2013;39(3):849.

113. Müller HJ, Heller D, Ziegler J. Visual search for singleton feature targets within and across feature dimensions. Perception \& psychophysics. 1995;57(1):1-17.

114. Müller HJ, Reimann B, Krummenacher J. Visual search for singleton feature targets across dimensions: Stimulus-and expectancy-driven effects in dimensional weighting. Journal of Experimental Psychology: Human perception and performance. 2003;29(5):1021. 115. Treisman AM, Gelade G. A feature-integration theory of attention. Cognitive psychology. 1980;12(1):97-136.

116. Gaspelin N, Luck SJ. Inhibition as a potential resolution to the attentional capture debate. Current opinion in psychology. 2019;29:12-8.

117. Cooke SF, Komorowski RW, Kaplan ES, Gavornik JP, Bear MF. Visual recognition memory, manifested as long-term habituation, requires synaptic plasticity in V1. Nature neuroscience. 2015;18(2):262.

118. Stokes MG. 'Activity-silent' working memory in prefrontal cortex: a dynamic coding framework. Trends in cognitive sciences. 2015;19(7):394-405.

119. Rao V, DeAngelis GC, Snyder LH. Neural correlates of prior expectations of motion in the lateral intraparietal and middle temporal areas. Journal of Neuroscience.

2012;32(29):10063-74.

120. Cosman JD, Lowe KA, Zinke W, Woodman GF, Schall JD. Prefrontal control of visual distraction. Current biology. 2018;28(3):414-20. e3.

121. Ipata AE, Gee AL, Gottlieb J, Bisley JW, Goldberg ME. LIP responses to a popout stimulus are reduced if it is overtly ignored. Nature neuroscience. 2006;9(8):1071.

122. Suzuki M, Gottlieb J. Distinct neural mechanisms of distractor suppression in the frontal and parietal lobe. Nature neuroscience. 2013;16(1):98.

123. Kusunoki M, Gottlieb J, Goldberg ME. The lateral intraparietal area as a salience map: the representation of abrupt onset, stimulus motion, and task relevance. Vision research. 2000;40(10-12):1459-68.

124. Thompson KG, Bichot NP. A visual salience map in the primate frontal eye field.

Progress in brain research. 2005;147:249-62.

125. Ogawa T, Komatsu $\mathrm{H}$. Target selection in area V4 during a multidimensional visual search task. Journal of Neuroscience. 2004;24(28):6371-82.

126. Heuer A, Schubö A. Cueing distraction: electrophysiological evidence for anticipatory active suppression of distractor location. Psychological research. 2019:1-11.

127. Hickey $\mathrm{C}$, Weaver $\mathrm{M}$, Kadel $\mathrm{H}$, van Zoest $\mathrm{W}$. How do we ignore salient distractors? Journal of Vision. 2017;17(10):205-.

128. Turatto $M$, Bonetti $F$, Pascucci $D$, Chelazzi L. Desensitizing the attention system to distraction while idling: A new latent learning phenomenon in the visual attention domain. Journal of Experimental Psychology: General. 2018;147(12):1827.

129. Mazaheri A, Jensen $O$. Rhythmic pulsing: linking ongoing brain activity with evoked responses. Frontiers in human neuroscience. 2010;4:177.

130. VanRullen R, Koch C. Is perception discrete or continuous? Trends in cognitive sciences. 2003; 7(5):207-13. 
131. Valera FJ, Toro A, John ER, Schwartz EL. Perceptual framing and cortical alpha rhythm. Neuropsychologia. 1981;19(5):675-86.

132. Busch NA, Dubois J, VanRullen R. The phase of ongoing EEG oscillations predicts visual perception. Journal of Neuroscience. 2009;29(24):7869-76.

133. Mathewson KE, Gratton G, Fabiani M, Beck DM, Ro T. To see or not to see: prestimulus $\alpha$ phase predicts visual awareness. Journal of Neuroscience. 2009;29(9):272532.

134. Van Diepen R, Foxe JJ, Mazaheri A. The functional role of alpha-band activity in attentional processing: The current zeitgeist and future outlook. Current opinion in psychology. 2019.

135. Samaha J, Bauer P, Cimaroli S, Postle BR. Top-down control of the phase of alphaband oscillations as a mechanism for temporal prediction. Proceedings of the National Academy of Sciences. 2015;112(27):8439-44.

136. van Diepen RM, Cohen MX, Denys D, Mazaheri A. Attention and temporal expectations modulate power, not phase, of ongoing alpha oscillations. Journal of cognitive neuroscience. 2015;27(8):1573-86.

137. VanRullen R, Zoefel B, Ilhan B. On the cyclic nature of perception in vision versus audition. Philosophical transactions of the Royal Society B: Biological sciences.

2014;369(1641):20130214.

138. Zanto TP, Rubens MT, Thangavel A, Gazzaley A. Causal role of the prefrontal cortex in top-down modulation of visual processing and working memory. Nature neuroscience. 2011;14(5):656.

139. Battistoni E, Stein T, Peelen MV. Preparatory attention in visual cortex. Annals of the New York Academy of Sciences. 2017;1396(1):92-107.

140. Posner MI. Orienting of attention. Quarterly journal of experimental psychology. 1980;32(1):3-25.

141. Liesefeld HR, Müller HJ. Distractor handling via dimension weighting. Current opinion in psychology. 2019.

142. Bae G-Y, Luck SJ. Dissociable decoding of spatial attention and working memory from EEG oscillations and sustained potentials. Journal of Neuroscience. 2018;38(2):409-22.

143. Smout CA, Tang MF, Garrido MI, Mattingley JB. Attention promotes the neural encoding of prediction errors. PLoS biology. 2019;17(2):e2006812.

144. Squire LR, Zola-Morgan S. The medial temporal lobe memory system. Science. 1991;253(5026):1380-6.

145. Eichenbaum H. A cortical-hippocampal system for declarative memory. Nature reviews neuroscience. 2000;1(1):41.

146. Kraus BJ, Robinson II RJ, White JA, Eichenbaum H, Hasselmo ME. Hippocampal "time cells": time versus path integration. Neuron. 2013;78(6):1090-101.

147. McGaugh JL. Memory--a century of consolidation. Science. 2000;287(5451):248-51.

148. O'Keefe J, Dostrovsky J. The hippocampus as a spatial map: preliminary evidence from unit activity in the freely-moving rat. Brain research. 1971.

149. Murray EA, Bussey TJ, Saksida LM. Visual perception and memory: a new view of medial temporal lobe function in primates and rodents. Annu Rev Neurosci. 2007;30:99122.

150. Kok P, Turk-Browne NB. Associative prediction of visual shape in the hippocampus. Journal of Neuroscience. 2018;38(31):6888-99. 
151. Seger CA. The basal ganglia in human learning. The Neuroscientist. 2006;12(4):28590.

152. Strumpf H, Mangun GR, Boehler CN, Stoppel C, Schoenfeld MA, Heinze HJ, et al. The role of the pulvinar in distractor processing and visual search. Human brain mapping. 2013;34(5):1115-32.

153. Nakajima M, Schmitt LI, Halassa MM. Prefrontal Cortex Regulates Sensory Filtering through a Basal Ganglia-to-Thalamus Pathway. Neuron. 2019.

154. Zelinsky GJ, Bisley JW. The what, where, and why of priority maps and their interactions with visual working memory. Annals of the New York Academy of Sciences. 2015;1339(1):154-64.

155. Sokolov EN. Higher nervous functions: The orienting reflex. Annual review of physiology. 1963;25(1):545-80.

156. Pascucci $D$, Turatto $M$. The distracting impact of repeated visible and invisible onsets on focused attention. Journal of experimental psychology: human perception and performance. 2015;41(3):879.

157. Turatto M, Pascucci D. Short-term and long-term plasticity in the visual-attention system: Evidence from habituation of attentional capture. Neurobiology of Learning and Memory. 2016;130:159-69.

158. Bonetti F, Turatto M. Habituation of oculomotor capture by sudden onsets: Stimulus specificity, spontaneous recovery and dishabituation. Journal of Experimental Psychology: Human Perception and Performance. 2019;45(2):264.

159. Turatto M, Bonetti F, Pascucci D. Filtering visual onsets via habituation: A contextspecific long-term memory of irrelevant stimuli. Psychonomic bulletin \& review.

2018;25(3):1028-34.

160. Thompson RF. Habituation: a history. Neurobiology of learning and memory. 2009;92(2):127.

161. Rankin CH, Abrams T, Barry RJ, Bhatnagar S, Clayton DF, Colombo J, et al. Habituation revisited: an updated and revised description of the behavioral characteristics of habituation. Neurobiology of learning and memory. 2009;92(2):135-8. 
Figures

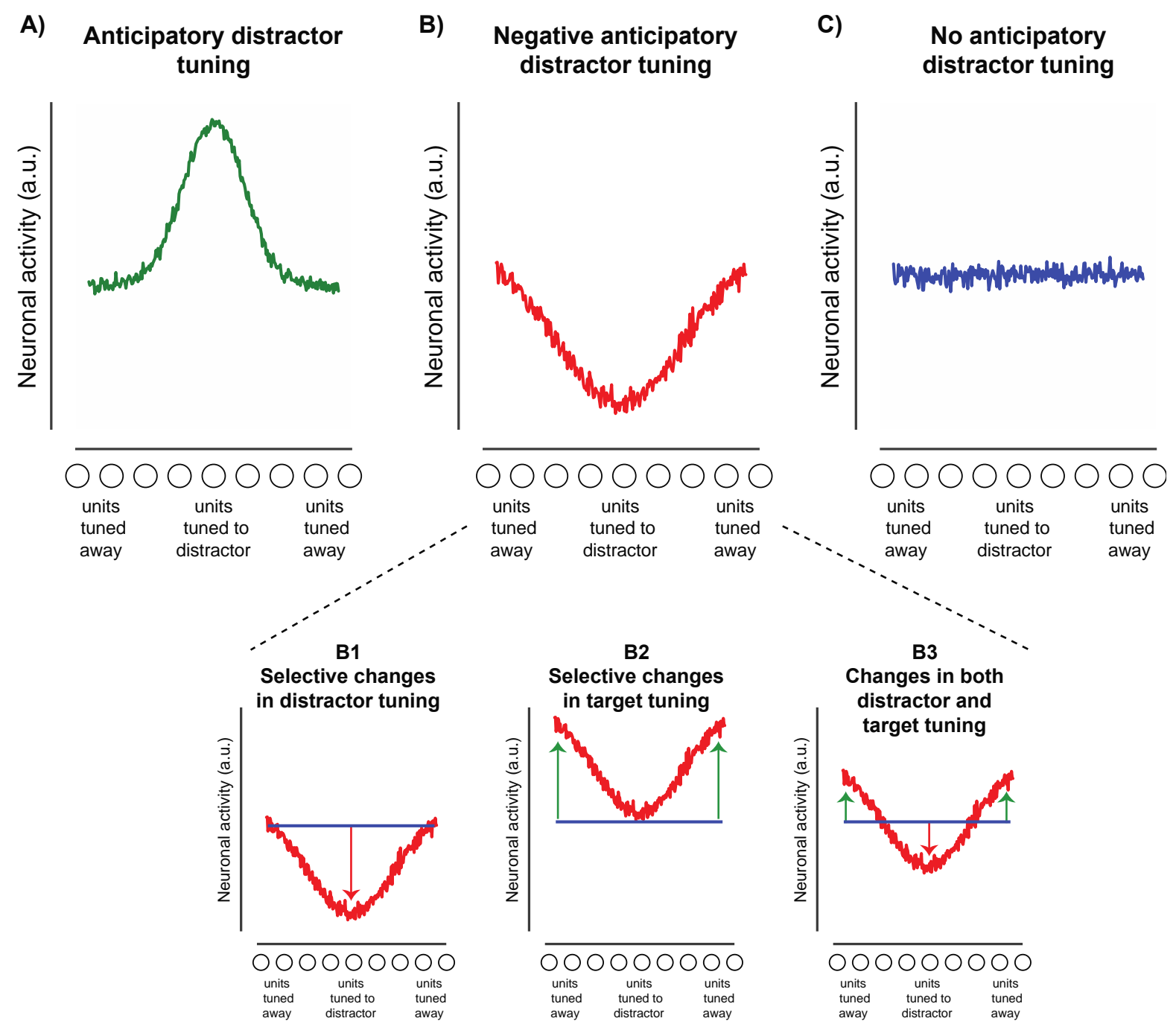




\section{A) Spatial tuning (total power)}

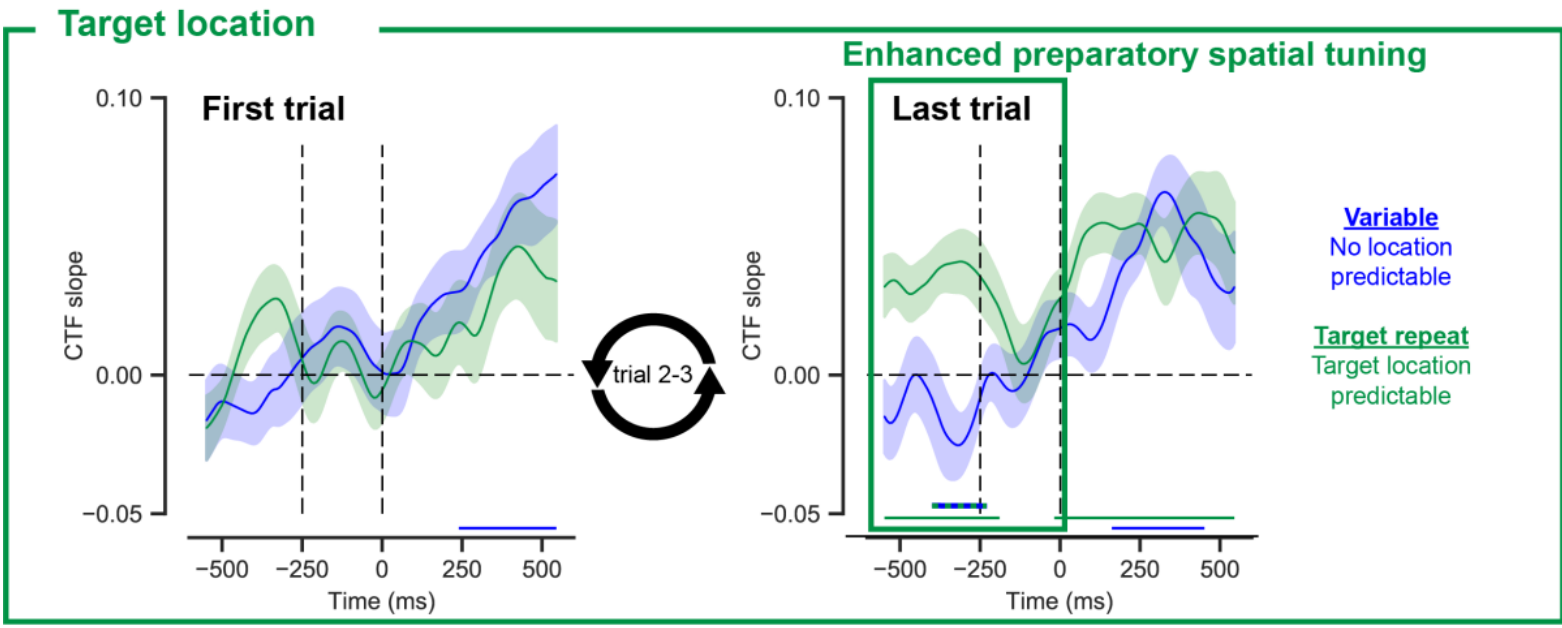

\section{Distractor location}
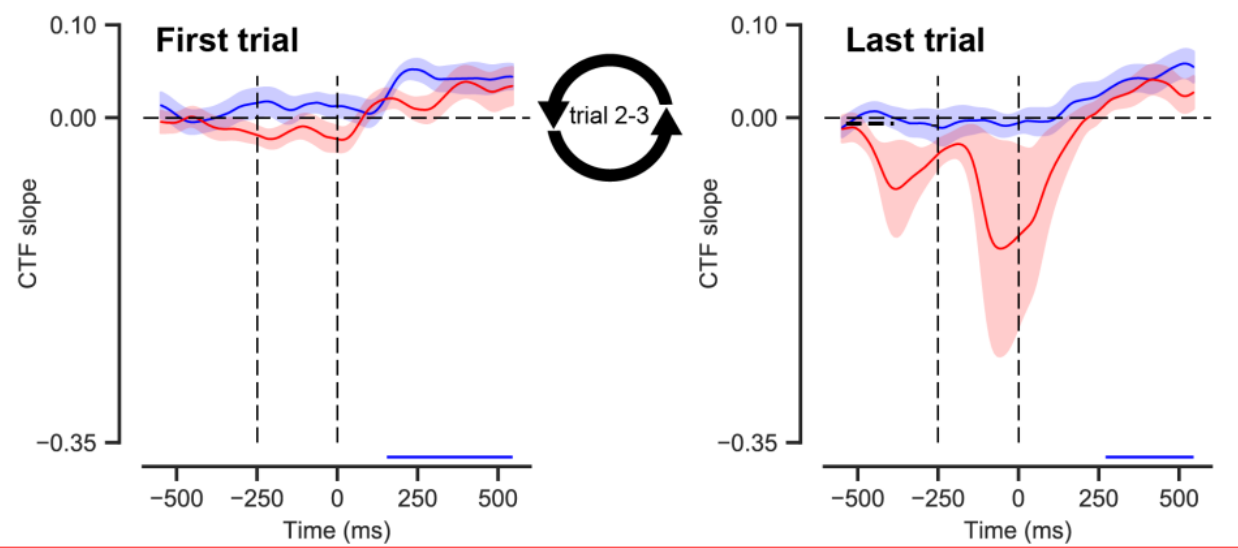

Distractor repeat Distractor location predictable

\section{B) Distractor elicited ERPs}

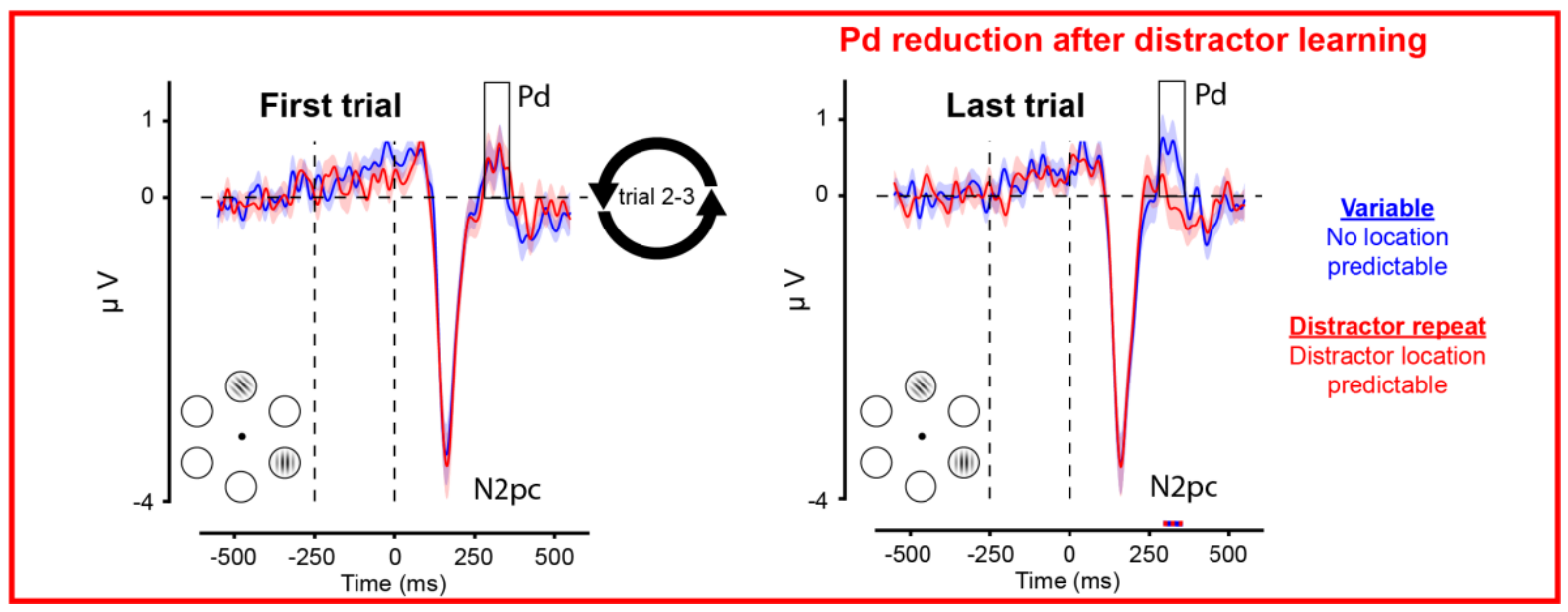




\section{Figure captions}

Figure 1 | This figure depicts three different scenarios of how expectations may modulate the representation of distracting information in anticipation of new sensory input (i.e., anticipatory distractor tuning). The tuning curves reflect selectivity of population-level neural activity to a particular feature (e.g., location or orientation). Expectations about upcoming distractor information may result in anticipatory tuning A) towards the expected distractor feature or B) away from the expected distractor feature resulting in negative anticipatory distractor tuning. The subplots of figure B illustrate different scenarios that may all produce negative tuning slopes: as consequence of reduced anticipatory tuning to the distractor (B1), of shifting anticipatory tuning away from the expected distractor to non-distractor features/locations (B2), or a combination of both (B3). The horizontal blue line in each subplot indicates the baseline situation of no expectation. C) Alternatively, distractor expectations may not be evident in anticipatory neural activity (firing) patterns.

Figure 2 |Summary of results of our recent EEG study (76) examining how distractor learning influences distractor processing. A) Slopes of channel tuning functions (CTF) tuned to the target location (top; green) and the distractor location (bottom; red), estimated based on total alpha power. While target location learning, induced by keeping the target location fixed across a block of trials, resulted in anticipatory tuning towards the expected target location, no such anticipatory tuning was observed after distractor location repetition. That is, no evidence was obtained for a change in anticipatory tuning to the distractor location in the last vs. the first trials of the block. B) Difference waveforms (contralateral - ipsilateral) revealing the N2pc and Pd elicited by distractors in the first trial and in the last trial of a block in which either the location of the distractor was repeated or varied across trials. As the figure shows, the Pd elicited by expected distractors (last trial distractorrepeat condition) was greatly reduced in amplitude compared to distractors that occurred at a non-predictable location (e.g., last trial variable condition). Double-colored thick lines in all plots indicate time points with a significant difference between the respective conditions after cluster correction $(p<0.05)$. 